\title{
Single-center experience of childhood Hodgkin lymphoma treated without radiotherapy
}

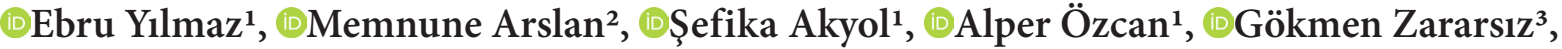

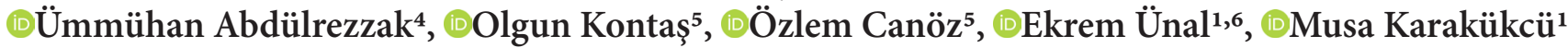 \\ ${ }^{1}$ Erciyes University, Faculty of Medicine, Department of Pediatrics, Division of Pediatric Hematology and Oncology \& Pediatric HSCT Unit, \\ Kayseri, Turkey \\ ${ }^{2}$ Erciyes University, Faculty of Medicine, Department of Pediatrics, Kayseri, Turkey \\ ${ }^{3}$ Erciyes University, Faculty of Medicine, Department of Biostatistics, Kayseri, Turkey \\ ${ }^{4}$ Erciyes University, Faculty of Medicine, Department of Nuclear Medicine, Kayseri, Turkey \\ ${ }^{5}$ Erciyes University, Faculty of Medicine, Department of Pathology, Kayseri, Turkey \\ ${ }^{6}$ Erciyes University Betül Ziya EREN Genome and Stem Cell, Department of Molecular Biology and Genetics, Kayseri, Turkey
}

Cite this article as: Yılmaz E, Arslan M, Akyol Ş, et al. Single-center experience of childhood Hodgkin lymphoma treated without radiotherapy. J Health Sci Med 2021; 4(6): 853-857.

\begin{abstract}
Aim: Hodgkin lymphoma (HL) constitutes $40 \%$ of childhood lymphomas and approximately $6 \%$ of all childhood cancers. It is tried to achieve cure with combined treatment modalities consisting of chemotherapy, radiotherapy, monoclonal antibodies, and new treatment agents such as nivolumab. Radiotherapy-related infertility, secondary cancer, thyroid dysfunction, cardiovascular diseases, pulmonary fibrosis, and local skin reactions can be seen in the pediatric age group with a long life expectancy. In this article, pediatric patients diagnosed with Hodgkin lymphoma without the use of radiotherapy in the treatment were evaluated retrospectively and the survival results were reported as a single-center experience.

Material and Method: The patients with Hodgkin Lymphoma in the Pediatrics Hematology-Oncology Center at Erciyes University between January 2010 and December 2019 were included in the study and the data of the patients were evaluated retrospectively.

Results: In 68 pediatric patients with a mean age of $10.7( \pm 4.6)$ years, the male/female ratio was 1.3 . The most detected finding at the time of diagnosis was cervical lymphadenopathy $(83.8 \%)$. The most common mixed cellular subtype was identified (48.5\%). Stage I-II disease was observed in $38.3 \%$ of the patients, and stage III-IV disease was observed in $61.7 \%$ of the patients. The median follow-up period of the patients was 61 (range, 8.3-161.6) months. Disease-free survival and overall survival were $85.3 \%$ and $94.1 \%$, respectively. Treatment modalities to be used in this disease group, which has a high chance of cure after cytotoxic treatment, should be selected considering treatment-related long-term complications.

Conclusion: Acceptable good results obtained without radiotherapy are satisfactory and the chance of curative success will increase with the addition of new target agents to the treatment.
\end{abstract}

Keywords: Hodgkin lymphoma, children, radiotherapy

\section{INTRODUCTION}

Hodgkin lymphoma (HL) constitutes $40 \%$ of childhood lymphomas and approximately $6 \%$ of all childhood cancers (1). Hodgkin lymphoma generally shows a bimodal distribution, mostly in young adults and older people. Its incidence peaks at 15-35 years of age and above 50 years of age (2). While the most common subtype in developed countries is the $60-70 \%$ nodular sclerosing type, mixed cellular type is most common in developing countries (3). It is among the malignant diseases with the best long-term outcome with $80-90 \%$ cure rate after chemotherapy. Relapsed or refractory to treatment is observed in $15-20 \%$ of patients (4). It is tried to achieve cure with combined treatment modalities consisting of chemotherapy, radiotherapy, monoclonal antibodies and new treatment agents such as nivolumomab. However, treatment-related side effects should not be ignored. While radiotherapy is an important part of treatment in adults with HL, the risk-benefit ratio in children is discussed. Radiotherapy-related infertility, secondary cancer, thyroid dysfunction, cardiovascular diseases, 
pulmonary fibrosis and local skin reactions can be seen in the pediatric age group with a long life expectancy $(2,3,5-7)$. In this article, pediatric patients diagnosed with Hodgkin lymphoma without the use of radiotherapy in the treatment were evaluated retrospectively and the survival results were reported as a single center experience.

\section{MATERIAL AND METHOD}

The study was carried out with the permission of Erciyes University Clinical Researchs Ethics Committee (Date: 11.12.2019, Decision No: 2019/854). All procedures were carried out in accordance with the ethical rules and the principles of the Declaration of Helsinki.

The patients younger than 18 years of age who were diagnosed with Hodgkin lymphoma in the Pediatrics Hematology-Oncology Center at Erciyes University between January 2010 and December 2019 were included in the study and the data of the patients were evaluated retrospectively. The data of the patients included in the study were obtained from the hospital electronic records and patient files.

In the study, demographic data of the patients, Hodgkin lymphoma subtype, presence of B symptoms, spread regions and stage of the disease, laboratory findings at the time of diagnosis, genetic study results, treatments and response to therapy, complications, relapse status, bone marrow transplantation and results, and prognostic factors were evaluated.

\section{Patients Characteristics and Chemotherapy Protocols}

The patients were diagnosed with HL by histopathological examination of biopsy samples. Bone marrow aspiration and biopsy, positron emission tomography/computed tomography (PET CT), and symptoms (fever, night sweats and weight loss) was used for staging of all patients. Before starting chemotherapy, the patients were staged according to Cotswolds modification of the Ann Arbor criteria (2).

Cyclophosphamide, vincristine, procarbazine, prednisone (COPP)/ doxorubicin, bleomycin, vinblastin, dacarbazine (ABVD) protocols were used alternately in the initial diagnosis. In early stage (Stage- $1 \mathrm{~A}$ and Stage2A) HL patients, 2 cycles of ABVD and 2 cycles of COPP protocols were started, and the number of cycles was rearranged to a maximum of 4 for each protocol, according to the response to treatment. In advanced stage (Stage IIB, Stage- 3 and Stage-4) HL patients, 3 cycles of ABVD and 3 cycles of COPP were started. According to the response to treatment, 4 th cycle ABVD and COPP were given. At least 2 cycles of ifosfamide, carboplatine, etoposide (ICE) protocol were given in refractory or relapsed patients.
Brentuximab was added to the treatment of refractory or relapsed patients diagnosed with HL after 2014. Autologous or allogeneic stem cell transplantation was performed in selected cases according to the underlying disease status such as immunodeficiency or relapse and refractory disease. No patient received radiotherapy.

\section{Statistical Analysis}

Descriptive statistics and quantitative variables were expressed as mean \pm standard deviation or median (minimum-maximum) according to whether the distributions were normal or not, using the Shapiro-Wilk test. Nominal variables were expressed as number of cases and percentage (\%). The Kaplan-Meier method was used to estimate survival probabilities and the log-rank test for comparisons.

\section{RESULTS}

A total of 68 patients, 38 males (56\%) and 30 females (44\%) diagnosed with HL, were included in the study. Demographic data of the patients are given in Table 1. While HL is 3.3 times more common in boys than girls in the $0-5$ age group, it is 1.5 times more common in boys in the 5-10 age group. In addition, the male-female ratio in patients older than 10 years is 0.9 .

\begin{tabular}{|lc|}
\hline Table 1. Patient characteristics & $\begin{array}{c}\text { Patient } \\
\text { Variable }\end{array}$ \\
\hline Median age (years) & $10.7( \pm 4.6)$ \\
Gender (male/female) & $38 / 30$ \\
Diagnosis of Patients & \\
Classical Hodgkin lymphoma (n=63) & \\
$\quad$ Nodular Sclerosis & $27(39.7 \%)$ \\
$\quad$ Mixed Cellularity & $33(48.5 \%)$ \\
$\quad$ Lymphocyte-rich & $2(2.9 \%)$ \\
$\quad$ Lymphocyte-depleted & $1(1.4 \%)$ \\
Nodular lymphocyte-predominant Hodgkin lymphoma & $1(1.4 \%)$ \\
Unknown subtype & $4(5.8 \%)$ \\
Diagnosis Staging & \\
$\quad$ Stage I & $5(7.3 \%)$ \\
\hline Stage II & $21(31 \%)$ \\
$\quad$ Stage III & $18(26.3 \%)$ \\
$\quad$ Stage IV & $24(35.4)$ \\
Disease Status & \\
\hline Complete remission 1 & $56(82.3 \%)$ \\
$\quad$ Relaps/refractory lymphoma & $12(17.6 \%)$ \\
\hline Notes: Values are expressed as n (\%). & \\
\hline
\end{tabular}

The majority of our patient group consisted of patients with stage III-IV (61.7\%). B symptoms were not observed in 45 patients. Figure 1 shows the ratio of B symptoms according to the stages of the patients. As lymph node, 
the most cervical lymph node involvement was observed (83.8\%); in the extra-lymphatic system, the most spread to bone/bone marrow was detected (35.3\%) (Table 2). Lymph node and non-lymph node involvements are summarized in Table 2. Bulky disease was detected in 10 patients (14.7\%) and vena cava superior syndrome and/or upper mediastinal syndrome was detected in 8 patients $(11.7 \%)$ at the time of admission. None of the patients had tumor lysis syndrome.

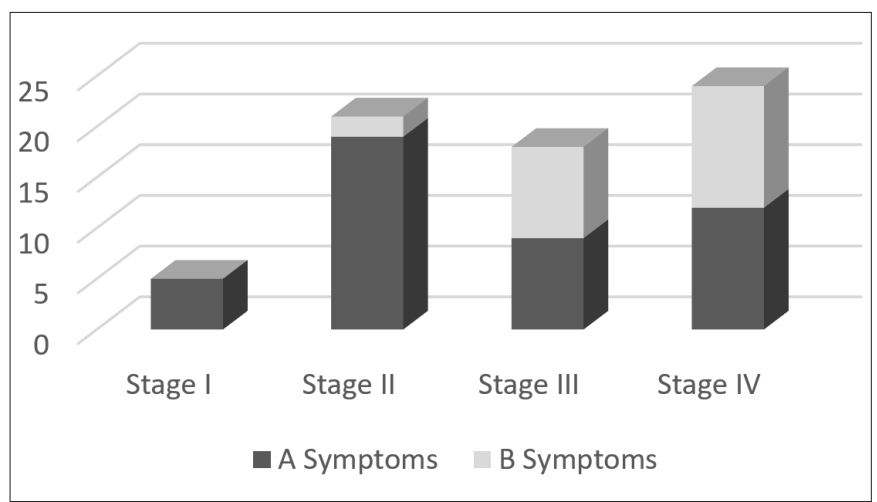

Figure 1. B Symptoms according to the stage of the patients

\begin{tabular}{|lc|}
\hline \multicolumn{2}{|l|}{ Table 2. Hodgkin lymphoma involvement sites } \\
\hline Site & Patient \\
\hline Cervical LN & $57(83.8 \%)$ \\
Mediastinal LN & $43(63.2 \%)$ \\
Axillary LN & $23(33.8 \%)$ \\
Paraaortic LN & $22(32.3 \%)$ \\
Supraclavicular LN & $19(27.9 \%)$ \\
Parailiac LN & $10(14.7 \%)$ \\
Submandibular LN & $7(10.2 \%)$ \\
Inguinal LN & $5(7.3 \%)$ \\
Waldeyer ring & $11(16.1)$ \\
Spleen & $23(33.8 \%)$ \\
Bone/bone marrow & $24(35.3 \%)$ \\
Lung & $10(14.7 \%)$ \\
Liver & $4(5.8 \%)$ \\
Intestine & $1(1.4 \%)$ \\
\hline Notes: Values are expressed as $n$ (\%). & \\
\hline
\end{tabular}

STAT-6 mutation in 2 patients, CD27 deficiency in 1 patient, F-BAR domain only protein 1 (FCHO1) mutation in 1 patient, Interleukin-2-inducible T-cell Kinase (ITK) mutation in 1 patient, phosphatidylinositol-4,5bisphosphate 3-kinase catalytic subunit delta (PIK3CD) mutation in 1 patient, Magnesium transporter 1 (MAGT1) deficiency, X-linked immunodeficiency with magnesium defect, Epstein-Barr virus (EBV) infection, and neoplasia (XMEN syndrome) in 1 patient were found in the genetic analysis of patients with relapse/refractory and reported in several publications (8).
Autologous hematopoietic stem cell transplantation was performed in 9 patients with relapse/refractory, and allogeneic hematopoietic stem cell transplantation was performed in 4 patients with immunodeficiency. One patient who was diagnosed with immunodeficiency and planned for allogeneic stem cell transplantation died due to sepsis. In addition, allogeneic stem cell transplantation is planned for 2 immunodeficiency patients.

The median follow-up period of the patients was 62 (range, 8.3-161.6) months. Disease-free (lymphoma, free) survival (DFS) and overall survival (OS) were $85.3 \%$ and $94.1 \%$, respectively (Figure 2 and 3 ). While DFS was $96.2 \%$ and OS was $100 \%$ in patients with stage I-II, DFS was $76.2 \%$ and OS was $90.5 \%$ in patients with stage IIIIV. One patient died of lymphoma progression, 2 patients died of sepsis after stem cell transplantation, and one patient died of sepsis secondary to immunodeficiency. Lymphoma-related mortality was $1.5 \%$ and overall mortality was $5.8 \%$.

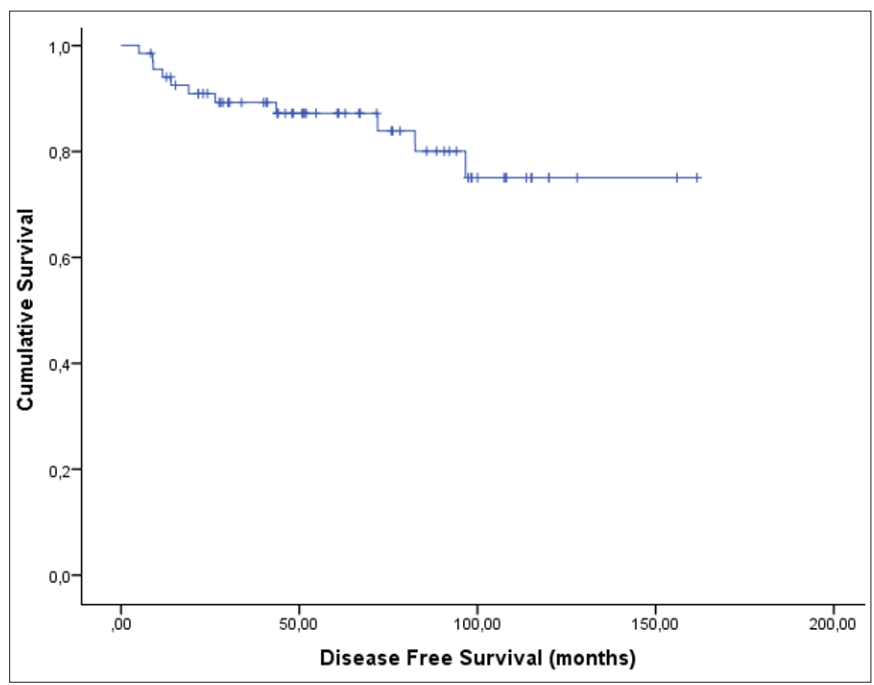

Figure 2. Disease-free survival graph of patients with Hodgkin lymphoma

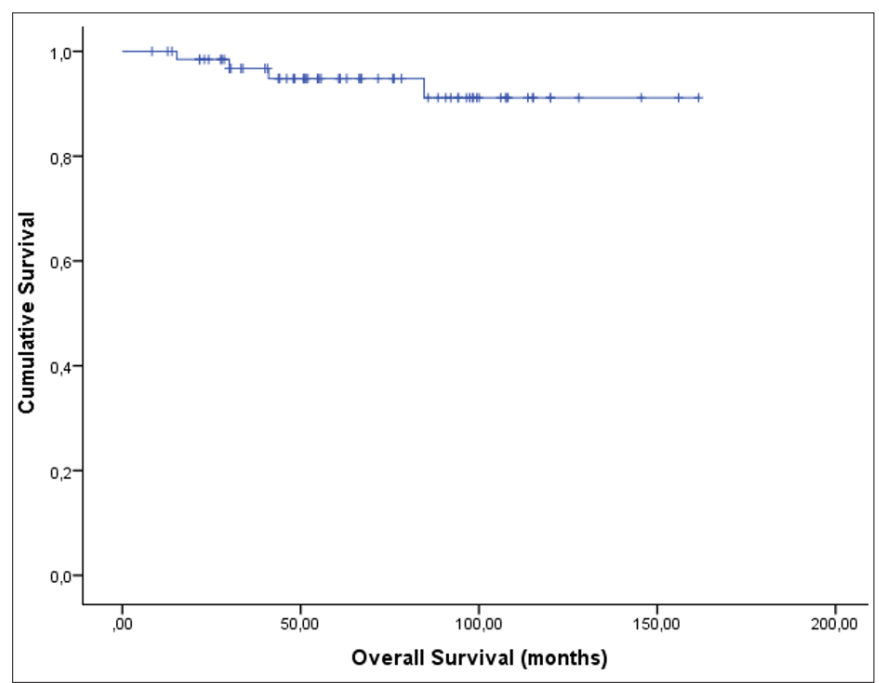

Figure 3. Overall survival graph of patients with Hodgkin lymphoma 


\section{DISCUSSION}

Hodgkin lymphoma is a malignant disease of unknown specific etiology characterized by enlargement of lymph nodes. Conditions such as genetic diseases, socioeconomic status, positive family history, and Epstein-Barr virus (EBV) infection increase the risk of developing HL $(2,9,10)$. In various studies, the age of incidence in the childhood age group is approximately 6-15.5 years. While Hodgkin lymphoma is more common between 5-10 years of age in developing countries, it has been found to be more common in children over the age of 10 in a study conducted in The United Kingdom (11-13). The mean age of the patients in our study was $10.7( \pm 4.6)$ years. $19.1 \%$ of the patients were aged $0-5$ years, $26.4 \%$ were aged $5-10$ years, and $54.5 \%$ were older than 10 years. The male/female ratio has been reported as $1.3-2.5(13,14) .56 \%$ of our patients were male and $44 \%$ were female. The male to female ratio was 1.26 , which is consistent with the literature. When we divide the patients into age groups, this ratio is; 3.3 in the $0-5$ age group; 1.5 in the $5-10$ age group, and 0.9 in patients older than 10 years old.

While nodular sclerosing type HL is observed at a rate of up to $80 \%$ in developed countries, mixed cellular type $\mathrm{HL}$ is observed at a rate of up to $60 \%$ in developing countries $(12,13,15)$. In our study, approximately half of the patients diagnosed with HL (48.5\%) were mixed cellular type, while the second most common (39.7\%) type was nodular sclerosing type and these findings were comparable with other studies conducted in our country $(16,17)$. The subtypes of the disease vary in relation to the development levels of the countries and the socioeconomic status of the people. Although the mixed cellular type was more common in our study, the incidence of nodular sclerosing type was seriously close to the incidence of the mixed cellular type. Generally, EBV is associated with mixed cellular HL lymphoma and is observed in young children, whereas nodular sclerosing type $\mathrm{HL}$ is more common in young adults and adolescents. Tumor cells are infected with EBV in $90 \%$ of cases in developing countries and $30 \%$ of cases in developed countries $(18,19)$. EBV positivity was detected in tumor cells in 27 of our patients (39.7\%).

Positron Emission Tomography (PET CT) is the standard imaging method for staging $\mathrm{HL}$ at the time of diagnosis and evaluating the response to treatment. However, it contains ionizing radiation. Nevertheless, in a study comparing magnetic resonance imaging and PET CT, PET CT was shown to be more sensitive in staging at the time of diagnosis and evaluating the response to treatment (20). We used PET CT for staging and evaluation of response to treatment in all of our patients.
Various combination chemotherapy regimens containing vinblastin, vincristine, dacarbazine, procarbazine, bleomycin, etoposide, cyclophosphamide, prednisone, doxorubicin, methotrexate are used in treatment (2). With the increase in survival rates, the focus is on eliminating or reducing the side effects that can be seen in long-term follow-ups. Studies are ongoing to reduce or eliminate anthracyclines due to its cardiotoxic effect, procarbazine for infertility, and radiotherapy due to its multiple side effects . In the studies of Dana Farber and St Jude Consortium, Children's Oncology Group (COG), and the German Society of Pediatric Oncology groups in low-risk patients who received radiotherapy, overall survival and eventfree survival were $96.1-100 \%$ and $86-95 \%$, respectively. In the studies of COG and the German Society of Pediatric Oncology group, overall survival and event-free survival were $93-98 \%$ and $82-94 \%$ in patients with medium and high-risk group HL who received radiotherapy (21). In a study using radiotherapy, in which patients with $43.1 \%$ early-stage disease and $55.9 \%$ advanced-stage disease, 5 -year overall survival, and event-free survival were $96.6 \%$ and $84.7 \%$ respectively (12). In another study in which early-stage 92 patients and advanced-stage 104 patients did not use radiotherapy, 5 -year overall survival and event-free survival were found to be $89.6 \%$ and $82.1 \%$, respectively (3). Mixed cellular lymphoma was the most common subtype in both studies $(3,12)$. In our study, $38.3 \%$ were evaluated as early-stage and $61.7 \%$ as advanced-stage. In the early and advanced patient group, 5-year overall survival is $90 \%$.

Autologous hematopoietic stem cell transplantation following high-dose chemotherapy is the standard treatment approach in relapsed and refractory patients. In the study, which included 38 pediatric patients with a diagnosis of $\mathrm{HL}, 10$-year survival and event-free-survival were $71.4 \%$ and $67.1 \%$, respectively (22). Allogeneic stem cell transplantation can be performed in relapsed cases after autologous stem cell transplantation or in HL that develops on the basis of disease such as immune deficiency $(9,10)$. In the meta-analysis, overall survival and relapsefree survival after transplantation were 50\% (41-58) and $31 \%$ (25-37) (21). One of our patients who underwent 10 autologous and 4 allogeneic stem cell transplants died due to post-transplant relapse and 2 died due to sepsis.

Although it varies depending on the stage of the disease in various studies, the total relapse rate has been reported as 11.2-30.4 $(11,12,23,24)$. Relapse is observed in $10 \%$ of patients with early stage $\mathrm{HL}$ and $25 \%$ in those with advanced stage HL after first-line treatment $(25,26)$. The relapse rate was found to be $14.7 \%$ in our patients. In relapsed and refractory cases, especially if the age of diagnosis is under 5 years, genetic studies are recommended to investigate diseases predisposing to lymphoma $(9,10)$. 


\section{CONCLUSION}

Treatment modalities to be used in this disease group, which has a high chance of cure after cytotoxic treatment, should be selected considering treatment-related longterm complications. Acceptable good results obtained without radiotherapy are satisfactory and the chance of curative success will increase with the addition of new target agents to the treatment.

\section{ETHICAL DECLARATIONS}

Ethics Committee Approval: The study was carried out with the permission of Erciyes University Clinical Researchs Ethics Committee (Date: 11.12.2019, Decision No: 2019/854).

Informed Consent: Because the study was designed retrospectively, no written informed consent form was obtained from patients.

Referee Evaluation Process: Externally peer-reviewed.

Conflict of Interest Statement: The authors have no conflicts of interest to declare.

Financial Disclosure: The authors declared that this study has received no financial support.

Author Contributions: All of the authors declare that they have all participated in the design, execution, and analysis of the paper, and that they have approved the final version.

Acknowledgment: The authors thank Prof. Dr. Mehmet Akif ÖZDEMİR, Prof. Dr. Türkan PATIROĞLU for their valuable contributions to the study.

\section{REFERENCES}

1. Zupanec S. Lymphoma. In: Tomlinson D, Kline NE, eds. Pediatric Oncology Nursing. Advanced Clinical Handbook. New York: Springer; 2010: 33-57.

2. Friedman D. Hodgkin Lymphoma. In: Lanzkowsky P, Lipton JM, Fish JD. Lanzkowsky's manual of pediatric hematology and oncology: Academic Press; 2016: 429-41.

3. Ashraf MS, Naz F, Yakoob MY. Characteristics and survival outcomes of children with Hodgkin lymphoma treated primarily with chemotherapy. J Pediatr Hematol Oncol 2019; 41: 452-6.

4. Cuccaro A, Bartolomei F, Cupelli E, Galli E, Giachelia M, Hohaus S. Prognostic factors in hodgkin lymphoma. Mediterr J Hematol Infect Dis 2014; 6: e2014053.

5. Dores GM, Metayer C, Curtis RE, et al. Second malignant neoplasms among long-term survivors of Hodgkin's disease: a populationbased evaluation over 25 years. J Clin Oncol 2002; 20: 3484-94.

6. Hancock SL, Donaldson SS, Hoppe RT. Cardiac disease following treatment of Hodgkin's disease in children and adolescents. J Clin Oncol 1993; 11: 1208-15.

7. Hancock SL, Cox RS, McDougall IR. Thyroid diseases after treatment of Hodgkin's disease. N Engl J Med 1991; 325: 599-605.

8. Patiroglu T, Haluk Akar H, Gilmour K, et al. A case of XMEN syndrome presented with severe auto-immune disorders mimicking autoimmune lymphoproliferative disease. Clin Immunol 2015; 159: 58-62.

9. Somekh I, Marquardt B, Liu Y, et al. Novel mutations in RASGRP1 are associated with immunodeficiency, immune dysregulation, and EBV-induced lymphoma. J Clin Immunol 2018; 38: 699-710.
10. Cansever M, Zietara N, Chiang SCC, et al. A rare case of activated phosphoinositide 3-kinase delta syndrome (APDS) presenting with hemophagocytosis complicated with hodgkin lymphoma. J Pediatr Hematol Oncol 2020; 42: 156-9.

11. Trehan A, Singla S, Marwaha RK, Bansal D, Srinivasan R. Hodgkin lymphoma in children: experience in a tertiary care centre in India. J Pediatr Hematol Oncol 2013; 35: 174-9.

12. Sherief LM, Elsafy UR, Abdelkhalek ER, et al. Hodgkin lymphoma in childhood: clinicopathological features and therapy outcome at 2 centers from a developing country. Medicine 2015; 94: 15.

13. Stoneham S, Ashley S, Pinkerton R, Hewitt M, Wallace W, Shankar AJEJoC. Hodgkin's lymphoma in children aged 5 years or lessThe United Kingdom experience. Eur J Cancer 2007; 43: 1415-21.

14. Hsu SC, Metzger ML, Hudson MM, et al. Comparison of treatment outcomes of childhood Hodgkin lymphoma in two US centers and a center in Recife, Brazil. Pediatr Blood Cancer 2007; 49: $139-44$.

15. Faizan M, Taj MM, Anwar S, et al. Comparison of presentation and outcome in 100 pediatric Hodgkin lymphoma patients treated at Children Hospital, Lahore, Pakistan and Royal Marsden Hospital, UK. J Coll Physician Surg Pak 2016; 26: 904-7.

16. Karhan AN, Varan A, Akyüz C, et al. Outcome of 102 patients under 5 years of age with Hodgkin lymphoma. Arch Argent Pediatr 2019; 117: 459-65.

17. Cavdar AO, Gozdasoglu S, Yavuz G, et al. Characteristics of "Early Type-I Pattern" (0-6 years) Hodgkin's Disease in Turkish Children. Turk J Haematol 2002; 19: 55-62.

18. Bazzeh F, Rihani R, Howard S, Sultan I. Comparing adult and pediatric Hodgkin lymphoma in the Surveillance, Epidemiology and End Results Program, 1988-2005: an analysis of 21734 cases. Leukemia \& lymphoma 2010; 51: 2198-207.

19. Naresh KN, Johnson J, Srinivas V, et al. Epstein-Barr virus association in classical Hodgkin's disease provides survival advantage to patients and correlates with higher expression of proliferation markers in Reed-Sternberg cells. Ann Oncol 2000; 11: 91-6.

20.Shapira-Zaltsberg G, Wilson N, et al. Whole-body diffusionweighted MRI compared to 18 FFDG PET/CT in initial staging and therapy response assessment of Hodgkin lymphoma in pediatric patients. Can Assoc Radiol J 2020; 71: 217-25.

21.Gómez-Almaguer D, González-Llano O, Jiménez-Antolinez V, Gómez-De León A. Treatment of classical Hodgkin's lymphoma in children and adolescents. Expert Opin Pharmacother 2019; 20: 1227-34.

22. Giulino-Roth L, O'Donohue T, Chen Z, et al. Outcome of children and adolescents with relapsed Hodgkin lymphoma treated with high-dose therapy and autologous stem cell transplantation: the memorial sloan kettering cancer center experience. Leuk Lymphoma 2018; 59: 1861-70.

23. Nachman JB, Sposto R, Herzog P, et al. Randomized comparison of low-dose involved-field radiotherapy and no radiotherapy for children with Hodgkin's disease who achieve a complete response to chemotherapy. J Clin Oncol 2002; 20: 3765-71.

24. Ali A, Sayed H, Farrag A, El-Sayed MJLr. Risk-based combinedmodality therapy of pediatric Hodgkin's lymphoma: A retrospective study. Leukemia Res 2010; 34: 1447-52.

25.Donaldson SS, Link MP, Weinstein HJ, et al. Final results of a prospective clinical trial with VAMP and low-dose involved-field radiation for children with low-risk Hodgkin's disease. J Clin Oncol 2007; 25: 332-7.

26. Schwartz CL, Constine LS, Villaluna D, et al. A risk-adapted, response-based approach using ABVE-PC for children and adolescents with intermediate- and high-risk Hodgkin lymphoma: the results of P9425. Blood 2009; 114: 2051-9. 